\title{
Risk Factors of Early Postoperative Small Bowel Obstruction Following a Proctectomy for Rectal Cancer
}

\author{
Jin Yong Shin \\ Department of Surgery, Haeundae Paik Hospital, Inje University College of Medicine, Busan, Korea
}

Purpose: Postoperative small bowel obstruction is a common and serious complication following a proctectomy, and early postoperative small bowel obstruction (EPSBO) leads to longer hospital stays, delays chemotherapy in advanced cases, and may be a contributor to mortality. The goal of this study is to identify the risk factors of EPSBO after a proctectomy for rectal cancer, thereby seeking to reduce the incidence of EPSBO.

Methods: Patients (735) who underwent a proctectomy for rectal cancer between March 2005 and February 2010 were entered into this study, and data were collected prospectively. Patients were judged to have EPSBO if, within the first 30 days, they presented symptoms such as nausea, vomiting and abdominal distention lasting for 2 days, and radiologic finding of small bowel obstruction after evidence of return of small bowel motility. The association between EPSBO and patients and surgery-related variables were studied by using univariate and multivariate analyses.

Results: EPSBO developed in 47 cases (6.4\%) and was the most frequently occurring complication in the early perioperative period following a proctectomy. The frequency of EPSBO according to operative variables shows that EPSBO developed in $3.0 \%$ of the patients who underwent laparoscopic surgery (LS) compared with $8.4 \%$ of the patients who underwent open surgery $(\mathrm{OS})(\mathrm{P}=0.004)$. OS (odds ratio $[\mathrm{OR}], 2.5)$ and a previous laparotomy $(\mathrm{OR}, 2.3)$ were independent risk factors for the development of EPSBO after a proctectomy for rectal cancer.

Conclusion: EPSBO is more likely to occur in patients who undergo OS or who have had a previous laparotomy. LS may be considered as a surgical procedure that can reduce the risk of EPSBO in patients undergoing a proctectomy for rectal cancer.

Keywords: Small bowel obstruction; Rectal neoplasms; Laparoscopy

\section{INTRODUCTION}

The rectum is the most common site of colorectal cancer and is affected in about $40 \%$ of cases [1-3]; thus, a proctectomy is a frequently performed surgical procedure. However, the proctectomy has a relatively high postoperative gastrointestinal complication rate (range, 10 to 15\%) due to anastomotic leakage or small bowel obstruction [4-6]. Particular attention should be paid to reduce

Received: June 26, 2011 Accepted: September 8, 2011

Correspondence to: Jin Yong Shin, M.D.

Department of Surgery, Haeundae Paik Hospital, Inje University College of

Medicine, 1435 Jwa 4-dong, Haeundae-gu, Busan 612-030, Korea

Tel: +82-51-742-7725, Fax: +82-51-797-0261

E-mail: jyong@medigate.net

(c) 2011 The Korean Society of Coloproctology

This is an open-access article distributed under the terms of the Creative Commons Attribution NonCommercial License (http://creativecommons.org/licenses/by-nc/3.0) which permits unrestricted non-

commercial use, distribution, and reproduction in any medium, provided the original work is properly cited. postoperative complications through identification of the risk factors involved and to improve the overall prognosis for rectal cancer.

Small bowel obstruction occurs in about $10 \%$ of patients after a curative resection of rectal cancer $[6,7]$ and is difficult to treat. In particular, early postoperative small bowel obstruction (EPSBO) developing within 4 weeks following surgery $[8,9]$ has been associated with increased hospital stays, postoperative adjuvant treatment delays, and mortality $[10,11]$. Nevertheless, little information is available regarding EPSBO other than the facts that it has a reported incidence of approximately $7 \%$ after a proctectomy [12, 13 ] and that rectal surgery and poor systemic conditions contribute to the risk of EPSBO $[12,13]$.

In multicenter prospective randomized trials that examined the short-term outcomes of laparoscopic and open colectomies, the laparoscopic colectomy was found to have the advantages of earlier postoperative recovery of bowel movement, shorter hospital stay, less postoperative pain, and less amount of intraoperative 
blood loss [14-16]. However, no significant difference was found between the two modalities in terms of the incidence of postoperative complications, and their comparative effects on morbidity have not been determined. In addition, prospective randomized studies $[14,15,17]$ have reported that the incidence of postoperative morbidity including EPSBO is similar for laparoscopic surgery and open surgery for colon cancer $[14,15]$ and that the incidences of EPSBO after these surgical modalities are not significantly different for rectal cancer [17]. However, small-scale prospective studies and meta-analyses [18-20] have suggested that laparoscopic surgery is superior to open laparotomy in terms of short-term outcomes, such as re-hospitalization, which indirectly may reflect EPSBO and postoperative morbidity rates and the duration of hospital stay. The purpose of this study was to determine the prevalence of EPSBO according to operative method and to identify the risk factors of EPSBO, which is one of the most common complications following a proctectomy for rectal cancer $[16,17]$.

\section{METHODS}

The study was conducted on 759 patients who underwent a proctectomy for rectal cancer with a tumor located below the sacral promontory (based on postoperative findings) from March 2005 to February 2010 at Pusan Paik Hospital. Twenty-two patients were excluded as follows: 11 underwent a synchronous gastrectomy or colectomy, 6 underwent salvage surgery for a recurrent lesion, and 5 underwent a proctectomy for metachronous rectal cancer. In addition, two patients that died on postoperative days 5 and 22 after surgery were excluded. Accordingly, 735 patients constituted the study cohort.

\section{Surgical technique}

The laparoscopic colectomy was adopted at the author's institution in December 2006. The procedure is performed by inserting a camera port using an open or closed method at the inferior edge of the umbilicus; subsequently, additional trocars are placed under laparoscopic vision (usually in the right upper abdomen, right lower abdomen, left upper abdomen, and left lower abdomen). Briefly, the operative procedure is as follows: Using a medial approach, the mesentery is scored at the sacral promontory, and the inferior mesenteric artery is identified early and ligated near its origin. The inferior mesenteric vein is then divided just below the pancreas for lymph-node dissection. For retroperitoneal dissection, the mesocolon and retroperitoneal tissues are detached via a medial approach. Mobilization of the splenic flexure is initiated by dividing the root of the transverse mesocolon along the pancreas. Parietal and omental attachments of the colon are then performed, and freeing of the splenic flexure is completed. Dissection of the rectum is performed along the anatomic space between the visceral and the parietal endopelvic fascia. In female patients, a traction suture is placed in the midportion of the uterus. In cases of middle and low rectal cancer, the rectum and its mesentery are dissected to the pelvic floor muscle to ensure en bloc resection of the total mesorectum. After the pelvic dissection has been completed, the distal end rectum is sectioned with one to three laparoscopic linear stapler(s). An incision of $5 \mathrm{~cm}$ is then made over the infraumbilical camera port site, and the bowel is exteriorized under a wound protector and divided with appropriate proximal clearance. After the anvil head of the circular stapler has been inserted into the end of the colon, the proximal colon is returned to the abdomen. Intracorporeal anastomosis under laparoscopic vision is performed as for open surgery. A diverting stoma is constructed when the anastomosis is problematic, for example, when the air-leak test is positive, the anastomosis is low lying, pelvic dissection is difficult, or bowel preparation is poor. For abdominoperineal resection, the sigmoid colon is divided, and the total mesorectal excision is performed using a laparoscopic technique. The perineal dissection is completed as for open surgery, and the specimen is extracted via the perineal wound. Reasons for conversion to open surgery included difficulties in exposition and tumor fixation to the pelvic sidewall. Expansion of the incision for extraction purposes was not considered as a conversion to open surgery.

Demographic information and details of surgical technique related variables and postoperative complications were obtained from a prospective database maintained. A patient was judged to have EPSBO if, within the first 30 days after proctectomy, he/she presented with symptoms such as nausea, vomiting, and abdominal distention of at least 2 days duration and radiologic finding of small bowel obstruction after evidence of return of small bowel motility.

Postoperative paralytic ileus was defined as no return of bowel function at more than six days after surgery and was not regarded as EPSBO. EPSBO duration was determined based on durations of symptoms and signs of EPSBO, regardless of the resumption of oral intake. Duration in patients with recurrent EPSBO, that is, after symptom and sign improvement, was defined as time between first symptom onset and date of improvement of symptoms and sign of recurrent EPSBO.

Perforation was defined as unintended perforation of a tumor or bowel during intraoperative manipulation. Patients were considered positive for intraoperative bleeding when a hemostatic maneuver, such as coagulation or ligature, failed to stop a hemorrhage, or when an additional procedure, such as gauze compression, was required for hemostasis. Previous abdominal surgery was considered present if a patient had previously undergone a laparotomy, including open appendectomy, but laparoscopic surgery was not considered as previous abdominal surgery. In terms of a lymphadenectomy, high ligation was defined as the ligation of the inferior mesenteric artery at its root whereas low ligation was defined as ligation below the origin of the left colic artery. The degree of bowel preparation was classified as 'adequate', 'inadequate', or not performed. Bowel preparation was considered inadequate when any non-prepararated liquid stool was observed. Anastomotic failure was considered any of the following clinical or radiologic find- 
ings: 1) pus or fecal discharge from the drain or surgical wound, 2) leakage of contrast through the anastomosis during water-soluble enema, and 3) the presence of an anastomotic defect based on digital rectal examination. A pelvic abscess was defined to be present when an imaging study showed fluid collection in the pelvic cavity of a patient with fever or leukocytosis in the absence of a finding of anastomotic failure.

\section{Statistics}

The statistical analysis was performed using SPSS ver. 12.0 (SPSS Inc., Chicago, IL, USA). The t-test was used to compare mean values, and the chi-square test or Fisher's exact test was used to detect the distribution difference of each group. A univariate logistic regression was used to identify risk factors of EPSBO, and factors with $\mathrm{P}<0.05$ were included in a multivariate analysis to identify the risk factors of EPSBO. Statistical significance was accepted for $\mathrm{P}<0.05$.

\section{RESULTS}

\section{Overall characteristics of the patients enrolled}

The median age of the 735 patients was 63.0 years (range, 28 to 90

Table 1. General characteristics of the patients with a proctectmy for rectal cancer

\begin{tabular}{|c|c|}
\hline Characteristics & Values \\
\hline Age (yr), median (range) & $63.0(28-90)$ \\
\hline Gender (male:female) & 426:309 \\
\hline Operative time (min), median (range) & $240.0(90-530)$ \\
\hline \multicolumn{2}{|l|}{ Type of resection, no (\%) } \\
\hline Anterior resection & $621(84.5)$ \\
\hline $\begin{array}{l}\text { Abdominoperineal resection or } \\
\text { Hartmann's proctosigmoidectomy }\end{array}$ & $114(15.5)$ \\
\hline \multicolumn{2}{|l|}{ Operative method, no (\%) } \\
\hline Laparoscopic surgery & $270(37.4)$ \\
\hline Open surgery & $465(62.6)$ \\
\hline \multicolumn{2}{|l|}{ T stage } \\
\hline$\leq 1$ & 79 \\
\hline 2 & 123 \\
\hline 3 & 476 \\
\hline 4 & 57 \\
\hline \multicolumn{2}{|l|}{ N stage (based on 6th ed. of TNM staging) } \\
\hline 0 & 439 \\
\hline 1 & 200 \\
\hline 2 & 96 \\
\hline \multicolumn{2}{|l|}{ Distant metastasis } \\
\hline Present & 63 \\
\hline Early postoperative small bowel obstruction & 47 \\
\hline
\end{tabular}

years); 426 were men and 309 were women. Laparoscopic surgery was performed in 270 patients, and conversion to open laparotomy was necessary in $5(1.8 \%)$. Because this study focused on actual treatment received rather than on intended treatment, 5 patients that underwent intra-operative conversion to open surgery were allocated to the open laparotomy group. In terms of TNM stages, 533 patients had a T3 tumor, 296 had lymph node metastasis, and 63 had distant metastasis. A sphincter-preserving proctectomy was performed in 621 patients (84.5\%), and open proctectomy in 465 (63.2\%), including the 5 conversions to open surgery (Table 1 ).

The median age of patients with EPSBO was significantly greater than that of patients without (65.1 vs. 61.9 years; $\mathrm{P}=0.043$ ). Patients that underwent an open proctectomy or a non-sphincter-preserving proctectomy, patients with a history of open laparotomy, and patients in which anti-adhesive was not applied had high incidences of EPSBO (Table 2).

\section{Postoperative surgical complications and EPSBO}

Overall, EPSBO occurred in 47 patients (6.4\%), and it was the most frequent complication during the early perioperative period. Of the other postoperative complications encountered, wound infec-

Table 2. Comparison of clinicopathologic characteristics between the patients with EPSBO and those with no EPSBO

\begin{tabular}{lccc}
\hline & $\begin{array}{c}\text { EPSB0 } \\
(\mathrm{n}=47)\end{array}$ & $\begin{array}{c}\text { No EPSB0 } \\
(\mathrm{n}=688)\end{array}$ & P-value \\
\hline Age (yr, median) & 65.13 & 61.92 & 0.043 \\
Male gender & $31(66.0)$ & $395(57.4)$ & 0.287 \\
Operative time (min, median) & 232.86 & 248.39 & 0.567 \\
ASA grading $\geq 3$ & $5(10.6)$ & $36(5.2)$ & 0.113 \\
BMI >25 & $8(17.0)$ & $189(27.4)$ & 0.128 \\
Advanced T stage (T3,4) & $31(66.0)$ & $502(73.0)$ & 0.312 \\
Nodal metastasis & $16(34.0)$ & $280(40.7)$ & 0.443 \\
Distant metastasis & $2(4.3)$ & $61(8.9)$ & 0.212 \\
Previous laparotomy & $16(34.0)$ & $123(17.9)$ & 0.011 \\
Protective stoma & $11(23.4)$ & $129(18.8)$ & 0.136 \\
Operative type (APR) & $14(30.0)$ & $100(14.5)$ & 0.011 \\
Open surgery & $39(83.0)$ & $426(61.9)$ & 0.004 \\
Combined operation & $3(6.4)$ & $61(8.9)$ & 0.399 \\
Intraoperative perforation & $7(14.9)$ & $70(10.2)$ & 0.212 \\
Intraoperative hemorrhage & $4(8.5)$ & $61(8.9)$ & 0.594 \\
High tie of IMA & $15(31.9)$ & $254(36.9)$ & 0.301 \\
Poor bowel preparation & $12(25.5)$ & $111(16.1)$ & 0.107 \\
Splenic flexure mobilization & $6(12.8)$ & $65(9.4)$ & 0.296 \\
No treatment with antiadhesive & $11(23.4)$ & $81(11.8)$ & 0.036 \\
\hline Val & & & \\
\hline
\end{tabular}

Values are presented as number (\%) unless otherwise specified.

EPSBO, early postoperative small bowel obstruction; ASA, American Society of Anesthesiologists; BMI, body mass index; APR, abdominoperineal resection; IMA, inferior mesenteric artery. 
Table 3. Surgical morbidity of patients with a proctectomy for rectal cancer in this study $(\mathrm{n}=621)$

\begin{tabular}{ll}
\hline Morbidity & No. (\%) \\
\hline Total early postoperative small bowel obstruction & $47(6.4)$ \\
Resolved with conservative management & 46 \\
Requiring operation & 1 \\
Wound infection $^{\text {Anastomotic events }}{ }^{\mathrm{a}}$ & $22(3.0)$ \\
Pelvic abscess $^{\mathrm{b}}$ & $15(2.4)$ \\
Rectovaginal fistula $^{\mathrm{c}}$ & $5(0.8)$ \\
Postoperative hemorrhage & $2(0.8)$ \\
\hline
\end{tabular}

${ }^{a}$ Anastomotic events included clinical anastomotic leakage and anastomotic defects identified at digital examination without clinical anastomotic leakage. ${ }^{\text {PPelvic }}$ abscess was defined as pelvic fluid collection without anastomotic defect and clinical symptoms such as fever and leukocytosis. ${ }^{~}$ Of 265 female patient who underwent an anterior resection.

tion was the most common (3.0\%), followed by anastomotic failure (2.4\%). EPSBO occurred at a mean of 8.4 days after surgery (range, 4 to 22 days), and the duration of EPSBO symptoms was 4 days (median value; range, 2 to 21 days). Of the 47 patients that developed EPSBO, 42 were diagnosed in hospital and 5 after discharge. Forty-six of the 47 improved after fasting, nasogastric tube insertion, and fluid therapy, and 15 patients required a water-soluble contrast imaging study (Gastrografin, Shering, Berlin, Germany); oral intake was resumed after studies. Two of the 46 patients with symptom or sign improvement as a result of conservative therapy were discharged 5 days and 7 days, respectively, after the same manner of treatment due to recurrent EPSBO on day 10 and day 14 following an index proctectomy, respectively. One patient that underwent open surgery required surgery to correct EPSBO at day 5 after index surgery after 3 days of conservative management, was discharged on day 11 after adhesiolysis, and experienced no subsequent event (Table 3).

\section{Incidence of EPSBO according to the surgical procedure}

The incidences of EPSBO after laparoscopic and sphincter-preserving surgery were $3.0 \%$ and $5.3 \%$, respectively, which were significantly lower than those after open and non-sphincter-preserving surgery ( $8.4 \%$ and $12.3 \% ; \mathrm{P}=0.004$ and $\mathrm{P}=0.011$, respectively). Of the 5 patients converted to open surgery during a laparoscopic proctectomy, 3 (60.0\%) subsequently developed EPSBO. Methods of constructing protective stoma were not significantly different in terms of the frequency of $\mathrm{EPSBO}(\mathrm{P}=0.136)$, but the incidence of EPSBO in the group with a protective stoma with an ileostomy was higher than it was in the group with a protective stoma with a colostomy $(\mathrm{P}=0.039)$. Other intra-operative complications, such as bleeding or perforation, were not found to be significantly associated with the occurrence of EPSBO (Table 4).
Table 4. Frequency of early postoperative small bowel obstruction (EPSBO) according to operative variables

\begin{tabular}{|c|c|c|}
\hline Variables & $\begin{array}{c}\text { No. of } \\
\text { EPSBO (\%) }\end{array}$ & P-value \\
\hline Operative maneuver & & 0.004 \\
\hline Laparoscopic surgery & $8(3.0)$ & \\
\hline Open surgery & $39(8.4)$ & \\
\hline Operative type & & 0.011 \\
\hline Anterior resection & $33(5.3)$ & \\
\hline $\begin{array}{l}\text { Abdominoperineal resection or } \\
\text { Hartmann's proctosigmoidectomy }\end{array}$ & $14(12.3)$ & \\
\hline Protective stoma ${ }^{a}$ & & $\begin{array}{c}0.136^{b}(0.108)^{c} \\
(0.039)^{d}(0.152)^{e}\end{array}$ \\
\hline Yes & $11(7.8)$ & \\
\hline Colostomy & $5(5.5)$ & \\
\hline Ileostomy & $6(12.0)$ & \\
\hline No & $22(4.6)$ & \\
\hline Intraoperative events $^{\dagger}$ & & 0.231 \\
\hline Yes & $12(8.6)$ & \\
\hline No & $35(5.8)$ & \\
\hline
\end{tabular}

${ }^{\mathrm{a}}$ Of 621 patients who an underwent anterior resection. ${ }^{\mathrm{b}} \mathrm{Compared}$ patients with protective stoma to those without protective stoma. 'Compared patients with colostomy as protective stoma to those without protective stoma. ${ }^{\mathrm{d} C o m p a r e d ~ p a t i e n t s ~}$ with ileostomy as protective stoma to those without protective stoma. ${ }^{e} \mathrm{Compared}$ patients with colostomy as protective stoma to those with protective stoma as ileostomy. IIntraoperative events included perforation and hemorrhage.

\section{Risk factors for EPSBO after proctectomy and association with post-operative complications}

Variables found to be potentially associated with EPSBO by the univariate analysis were age, previous history of a laparotomy, nonsphincter preserving surgery, an open proctectomy, and no application of anti-adhesives. The multivariate analysis revealed that an open proctectomy (odds ratio [OR], 2.46; 95\% confidence interval [CI], 1.06 to $5.35 ; \mathrm{P}=0.035)$ and a history of a previous laparotomy (OR, 2.29; 95\% CI, 1.18 to $4.46 ; \mathrm{P}=0.015$ ) were independent risk factors of EPSBO after a proctectomy. Significant differences in the risks of developing EPSBO were observed between patients that underwent a sphincter-preserving proctectomy with major post-operative complications, such as anastomotic failure, pelvic abscess, and postoperative bleeding, and those that did not develop a postoperative complication $(24.0 \%$ vs. $4.5 \%$; $\mathrm{P}=0.001)$ (Tables 5 and 6).

\section{DISCUSSION}

Although anastomotic leakage, which has an incidence of $10 \%$, is the most common complication after a proctectomy $[7,16,21]$, EPSBO has been reported to be the second most common complication $[7,22]$. Nevertheless, few studies have investigated EPSBO 
Table 5. Risk factors for EPSBO after a proctectomy in rectal cancer

\begin{tabular}{|c|c|c|c|c|c|c|}
\hline \multirow{2}{*}{ Variables } & \multicolumn{3}{|c|}{ Univariate analysis } & \multicolumn{3}{|c|}{ Multivariate analysis } \\
\hline & $\mathrm{OR}$ & $95 \% \mathrm{Cl}$ & P-value & $\mathrm{OR}$ & $95 \% \mathrm{Cl}$ & P-value \\
\hline Age (yr) & 1.03 & $1.00-1.06$ & 0.044 & 1.02 & $0.99-1.05$ & 0.132 \\
\hline Male gender & 1.44 & $0.77-2.68$ & 0.253 & & & \\
\hline ASA grading $\geq 3$ & 2.16 & $0.80-5.78$ & 0.126 & & & \\
\hline $\mathrm{BMI}>25$ & 0.54 & $0.25-1.18$ & 0.123 & & & \\
\hline Advanced T stage $(T 3,4)$ & 0.72 & $0.38-1.34$ & 0.300 & & & \\
\hline Nodal metastasis & 0.75 & $0.40-1.40$ & 0.369 & & & \\
\hline Distant metastasis & 0.46 & $0.11-1.93$ & 0.286 & & & \\
\hline Previous laparotomy & 2.37 & $1.26-4.47$ & 0.008 & 2.29 & $1.18-4.46$ & 0.015 \\
\hline Operative type (APR vs. AR) & 2.50 & $1.29-4.83$ & 0.007 & 1.71 & $0.73-4.00$ & 0.216 \\
\hline Protective stoma & 1.74 & $0.82-3.68$ & 0.147 & & & \\
\hline Open surgery & 2.78 & $1.27-6.08$ & 0.010 & 2.46 & $1.06-5.35$ & 0.035 \\
\hline Combined operation & 0.70 & $0.21-2.32$ & 0.561 & & & \\
\hline Intraoperative perforation & 1.55 & $0.67-3.58$ & 0.310 & & & \\
\hline Intraoperative hemorrhage & 0.95 & $0.33-2.75$ & 0.929 & & & \\
\hline High tie of IMA & 0.80 & $0.43-1.51$ & 0.492 & & & \\
\hline Poor bowel preparation & 1.77 & $0.89-3.52$ & 0.102 & & & \\
\hline Splenic flexure mobilization & 1.40 & $0.57-3.43$ & 0.458 & & & \\
\hline No treatment with antiadhesive & 2.29 & $1.12-4.68$ & 0.023 & 1.47 & $0.59-3.65$ & 0.412 \\
\hline
\end{tabular}

EPSBO, early postoperative small bowel obstruction; OR, odds ratio; Cl, confidence interval; ASA, American Society of Anesthesiologists; BMl, body mass index; APR, abdominoperineal resection; AR, anterior resection; IMA, inferior mesenteric artery.

Table 6. Relationship between the incidence of EPSBO and postoperative surgical complications in patients who underwent anterior resection

\begin{tabular}{lcr}
\hline Variables & EPSB0 (n, \%) & P-value \\
\hline Patients with postoperative surgical event & $6(24.0)$ & $0.001^{*}$ \\
Patients without postoperative surgical event & $27(4.5)$ & \\
\hline
\end{tabular}

EPSBO, early postoperative small bowel obstruction.

*P-value is calculated from Fisher's exact test.

after a proctectomy; thus, information regarding EPSBO is derived indirectly from reports on EPSBO after a colectomy for colorectal cancer $[12,13]$. Because the present study includes all patients with rectal cancer, defined as a tumor located below the sacral promontory based on intraoperative findings, its results provide, for the first time, an estimate of the incidence and of the risk factors for EPSBO following a proctectomy in rectal cancer. In the present study, the rate of EPSBO after a proctectomy was $6.4 \%$, which is similar to the rate of 6-7\% reported by Poon et al. [7] and Nakajima et al. [12].

In the present study, based on a multivariate analysis, open surgery and a previous history of laparotomy were found to be risk factors of EPSBO after rectal surgery. This result concurs with the previous suggestion that a laparotomy increases the postoperative small bowel obstruction rate [23]. The reason for the high rate of EPSBO after an open proctectomy seems to be explained by the findings of a previous study on EPSBO after a colectomy [12]; that is, open surgery damages the abdominal walls and small bowel manipulation during surgery increases local and/or systemic inflammatory reactions [24]. With regard to the advantages of a laparoscopic proctectomy, a small prospective randomization study $[18]$ and a meta-analysis $[19,20]$ both showed that it reduces adhesion-related morbidities and produces better short-term results $[19,20]$. In addition, in the present study, the rate of EPSBO after a proctectomy was found to be lower in the laparoscopic group. These findings support the superiority of laparoscopic surgery with respect to perioperative short-term outcomes and may provide clues regarding performance of the laparoscopic surgery for rectal cancer. In addition, the present study shows that a history of laparotomy is a risk factor EPSBO after a proctectomy. However, it is difficult to propose a mechanism or draw any conclusion about the relation between a history of laparotomy and EPSBO after a proctectomy because of the lack of available information. However, it seems likely that the higher rate of EPSBO in cases with a history of laparotomy is related to the severity of adhesion, which is in-line with previous suggestions that postoperative adhesion was increased more in the patients with a previous history of laparotomy [7, 25, 26]. However, one report suggested that previous 
abdominal surgery does not increase the incidence of small bowel obstruction in patients with ulcerative colitis [27].

It is interesting that, based on the univariate analysis in the present study, the rate of EPSBO after non-sphincter-preserving proctectomy was higher than it was after a sphincter-preserving proctectomy. Previous studies $[25,28]$ suggested that internal herniation into a peritoneal defect or into the peristomal lateral space is the likely mechanism of EPSBO after a non-sphincter-preserving proctectomy. The reported rates of EPSBO after a non-sphincterpreserving proctectomy fall in the range $1.8-3.2 \%$ [25]; thus, the rate of $12.3 \%$ in the present study was quite high. In this study, the risk factors of EPSBO were analyzed in patients that underwent an abdominoperineal resection or Hartmann's procedure, but no meaningful findings were obtained with respect to identification of risk factors (data not shown). It appears the relatively small number of 114 patients that underwent a non-sphincter-preserving proctectomy prevented the identification of any significant risk factor for EPSBO in this subgroup, which indicates that a larger prospective study is required to increase understanding of EPSBO after a non-sphincter-preserving proctectomy.

In terms of the influence of a protective stoma on the frequency of EPSBO after a proctectomy, the rate of EPSBO was found to be higher in patients that underwent an ileostomy as a protective stoma after a sphincter-preserving proctectomy, which concurs with the result of Poon et al. [7] that the possibility of EPSBO was higher in patients that underwent a sphincter-preserving proctectomy with an ileostomy being performed as a protective stoma. In the present study, the incidence of EPSBO tended to be higher in patients who underwent an ileostomy as a protective stoma after sphincterpreserving proctectomy than in patients who underwent a colostomy as a protective stoma after sphincter-preserving proctectomy. Although this result was not of statistical significance, the use of an ileostomy as a preferred mode of protective stoma following sphincter-preserving proctectomy should be re-evaluated in view of the high incidence of EPSBO after a proctectomy. However, this possibility should be clarified by a larger scale study.

The $24 \%$ higher incidence of EPSBO among patients that experienced a postoperative surgical complication, such as anastomotic failure or a pelvic abscess, implies an association between EPSBO and surgical complications after a sphincter-preserving proctectomy. This finding is consistent with that of a previous paper, in which intraperitoneal inflammation was found to be a cause of EPSBO after a laparotomy [25]. Accordingly, surgically related complications may play a role in the development of EPSBO; thus, because EPSBO may delay the initiation of chemotherapy or prolong the hospital stay, particular attention is required to reduce surgically related complications. Therefore, reductions in the frequency of EPSBO potentially would serve to improve the overall prognosis in rectal cancer.

EPSBO after a proctectomy was found to be associated with an open proctectomy and a history of laparotomy. The findings of the present study suggest that a laparoscopic proctectomy has a lower incidence of EPSBO than an open proctectomy.

\section{CONFLICT OF INTEREST}

No potential conflict of interest relevant to this article was reported.

\section{REFERENCES}

1. Ju JH, Chang SC, Wang HS, Yang SH, Jiang JK, Chen WC, et al. Changes in disease pattern and treatment outcome of colorectal cancer: a review of 5,474 cases in 20 years. Int J Colorectal Dis 2007; 22:855-62.

2. Iversen LH, Norgaard M, Jepsen P, Jacobsen J, Christensen MM, Gandrup P, et al. Trends in colorectal cancer survival in northern Denmark: 1985-2004. Colorectal Dis 2007;9:210-7.

3. Birgisson H, Talback M, Gunnarsson U, Pahlman L, Glimelius B. Improved survival in cancer of the colon and rectum in Sweden. Eur J Surg Oncol 2005;31:845-53.

4. Paun BC, Cassie S, MacLean AR, Dixon E, Buie WD. Postoperative complications following surgery for rectal cancer. Ann Surg 2010;251:807-18.

5. Marusch F, Koch A, Schmidt U, Wenisch H, Ernst M, Manger T, et al. Early postoperative results of surgery for rectal carcinoma as a function of the distance of the tumor from the anal verge: results of a multicenter prospective evaluation. Langenbecks Arch Surg 2002;387:94-100.

6. Zaheer S, Pemberton JH, Farouk R, Dozois RR, Wolff BG, Ilstrup D. Surgical treatment of adenocarcinoma of the rectum. Ann Surg 1998;227:800-11.

7. Poon JT, Law WL, Chu KW. Small bowel obstruction following low anterior resection: the impact of diversion ileostomy. Langenbecks Arch Surg 2004;389:250-5.

8. Ellozy SH, Harris MT, Bauer JJ, Gorfine SR, Kreel I. Early postoperative small-bowel obstruction: a prospective evaluation in 242 consecutive abdominal operations. Dis Colon Rectum 2002;45: 1214-7.

9. Schein M, Sajja SB, Yenumula PR. Early postoperative intestinal obstruction. Curr Surg 2002;59:289-95.

10. Schulz U, Drüner HU, Lang HJ. Analysis of postoperative deaths in colon and rectal cancer. Langenbecks Arch Chir 1977;343:195-204.

11. Longo WE, Virgo KS, Johnson FE, Oprian CA, Vernava AM, Wade TP, et al. Risk factors for morbidity and mortality after colectomy for colon cancer. Dis Colon Rectum 2000;43:83-91.

12. Nakajima J, Sasaki A, Otsuka K, Obuchi T, Nishizuka S, Wakabayashi G. Risk factors for early postoperative small bowel obstruction after colectomy for colorectal cancer. World J Surg 2010; 34:1086-90.

13. Shin JY, Hong KH. Risk factors for early postoperative small-bowel obstruction after colectomy in colorectal cancer. World J Surg 2008;32:2287-92.

14. Veldkamp R, Kuhry E, Hop WC, Jeekel J, Kazemier G, Bonjer HJ, et al. Laparoscopic surgery versus open surgery for colon cancer: 
short-term outcomes of a randomised trial. Lancet Oncol 2005;6: 477-84.

15. Clinical Outcomes of Surgical Therapy Study Group. A comparison of laparoscopically assisted and open colectomy for colon cancer. N Engl J Med 2004;350:2050-9.

16. Guillou PJ, Quirke P, Thorpe H, Walker J, Jayne DG, Smith AM, et al. Short-term endpoints of conventional versus laparoscopicassisted surgery in patients with colorectal cancer (MRC CLASICC trial): multicentre, randomised controlled trial. Lancet 2005; 365:1718-26.

17. Kang SB, Park JW, Jeong SY, Nam BH, Choi HS, Kim DW, et al. Open versus laparoscopic surgery for mid or low rectal cancer after neoadjuvant chemoradiotherapy (COREAN trial): short-term outcomes of an open-label randomised controlled trial. Lancet Oncol 2010;11:637-45.

18. Ng SS, Leung KL, Lee JF, Yiu RY, Li JC, Hon SS. Long-term morbidity and oncologic outcomes of laparoscopic-assisted anterior resection for upper rectal cancer: ten-year results of a prospective, randomized trial. Dis Colon Rectum 2009;52:558-66.

19. Gao F, Cao YF, Chen LS. Meta-analysis of short-term outcomes after laparoscopic resection for rectal cancer. Int J Colorectal Dis 2006;21:652-6.

20. Aziz O, Constantinides V, Tekkis PP, Athanasiou T, Purkayastha S, Paraskeva $P$, et al. Laparoscopic versus open surgery for rectal cancer: a meta-analysis. Ann Surg Oncol 2006;13:413-24.

21. Lelong B, Bege T, Esterni B, Guiramand J, Turrini O, Moutardier V, et al. Short-term outcome after laparoscopic or open restorative mesorectal excision for rectal cancer: a comparative cohort study. Dis Colon Rectum 2007;50:176-83.

22. Vignali A, Fazio VW, Lavery IC, Milsom JW, Church JM, Hull TL, et al. Factors associated with the occurrence of leaks in stapled rectal anastomoses: a review of 1,014 patients. J Am Coll Surg 1997; 185:105-13.

23. Duepree HJ, Senagore AJ, Delaney CP, Fazio VW. Does means of access affect the incidence of small bowel obstruction and ventral hernia after bowel resection? Laparoscopy versus laparotomy. J Am Coll Surg 2003;197:177-81.

24. Kalff JC, Carlos TM, Schraut WH, Billiar TR, Simmons RL, Bauer AJ. Surgically induced leukocytic infiltrates within the rat intestinal muscularis mediate postoperative ileus. Gastroenterology 1999; 117:378-87.

25. Sajja SB, Schein M. Early postoperative small bowel obstruction. Br J Surg 2004;91:683-91.

26. Nieuwenhuijzen M, Reijnen MM, Kuijpers JH, van Goor H. Small bowel obstruction after total or subtotal colectomy: a 10-year retrospective review. Br J Surg 1998;85:1242-5.

27. Dolejs S, Kennedy G, Heise CP. Small bowel obstruction following restorative proctocolectomy: affected by a laparoscopic approach? J Surg Res 2011;170:202-8.

28. Sannella NA. Early and late obstruction of the small bowel after abdominoperineal resection. Am J Surg 1975;130:270-2. 